\title{
Missing heritability in genome-wide association study research
}

\section{Paolo Vineis and Neil Pearce}

Much of the literature on genome-wide association (GWA) studies is based on the premise that an important proportion of common diseases is heritable, and that this proportion is likely to be due to detectable genetic variants (Missing heritability and strategies for finding the underlying causes of complex disease. Nature Rev.
Genet. 11, 446-450 (2010)) $)^{1,2}$. There is a wide gap between the population variation in disease explained by the results of GWA studies (usually less than 10\%) and estimates of heritability (often more than 50\%). However, population variation should not be confused with the proportion of disease explained by an exposure or

\begin{tabular}{|c|c|c|c|c|c|}
\hline \multicolumn{2}{|l|}{ Exposure } & \multicolumn{2}{|c|}{ Genetic variant } & \multicolumn{2}{|c|}{ Attributable risk } \\
\hline Relative risk & $\begin{array}{l}\% \text { cases } \\
\text { exposed }\end{array}$ & Relative risk & $\begin{array}{l}\% \text { cases } \\
\text { exposed }\end{array}$ & Exposure & $\begin{array}{l}\text { Genetic } \\
\text { variant }\end{array}$ \\
\hline \multirow[t]{9}{*}{10.0} & $90 \%$ & 10.0 & $90 \%$ & $81 \%$ & $81 \%$ \\
\hline & $90 \%$ & & $0 \%$ & $81 \%$ & $0 \%$ \\
\hline & $50 \%$ & & $50 \%$ & $45 \%$ & $45 \%$ \\
\hline & $80 \%$ & & $20 \%$ & $72 \%$ & $18 \%$ \\
\hline & $90 \%$ & 2.0 & $90 \%$ & $81 \%$ & $45 \%$ \\
\hline & $90 \%$ & & $0 \%$ & $81 \%$ & $0 \%$ \\
\hline & $50 \%$ & & $50 \%$ & $45 \%$ & $25 \%$ \\
\hline & $80 \%$ & & $20 \%$ & $72 \%$ & $10 \%$ \\
\hline & $20 \%$ & & $80 \%$ & $18 \%$ & $40 \%$ \\
\hline
\end{tabular}

*Attributable risks in the population as a function of relative risk and proportion of exposed cases, under the assumption of a linear combination of genes and environment. The attributable risk is based on Miettinen's formula for case-control studies.

trait $^{3}$. For example, phenylketonuria (PKU) can be avoided either by removing the PKU gene mutations from the population or by a low dietary phenylalanine. Thus, $100 \%$ of cases would be prevented either by removing the mutation or by adopting a low phenylalanine diet. In a population in which everyone has a high phenylalanine diet, the condition will appear to be $100 \%$ genetic; in a population in which everyone has the mutation, the condition will appear to be $100 \%$ environmental. Thus, the genetic and environmental components are inseparable. In fact, as we learn more about a particular disease, it is inevitable that the attributable proportions for different risk factors will sum to more than $100 \%$ (see examples in TABLE 1), whereas the proportions of population variation cannot add up to more than $100 \%$.

Paolo Vineis is at the MRC/HPA Centre for Environment and Health, School of Public Health, Imperial College, London; and the Human Genetics Foundation, Torino, Italy.

Neil Pearce is at the Centre for Public Health Research Massey University Wellington Campus, Private BOX 756, Wellington, New Zealand.

Correspondence to P.V. e-mail:p.vineis@imperial.ac.uk

doi: 10.1038/nrg2809-c2

1. Eichler, E. E. et al. Missing heritability and strategies for finding the underlying causes of complex disease. Nature Rev. Genet. 11, 446-450 (2010).

2. Manolio, T. A. et al. Finding the missing heritability of complex diseases. Nature 461, 747-753 (2009).

3. Lewontin, R. C. The analysis of variance and the analysis of causes. Am. J. Hum. Genet. 26, 400-411 (1974). 